\author{
Acta Scientifica Naturalis \\ Former Annual of Konstantin Preslavsky University - Chemistry, Physics, Biology, Geography \\ Journal homepage: http://www.shu.bg
}

Received: 30.10 .2016

Accepted: 11.01.2017

\title{
Electronic setup for fluorescence emission measurements and long-time constant-temperature maintenance of Single-Walled Carbon Nano-Tubes in water solutions
} \author{
Matteo De Rosa ${ }^{1}$, Laura De Nardo ${ }^{1,2}$, Michele Bello ${ }^{1,3}$, Nikolay Uzunov ${ }^{3,4}$ \\ ${ }^{1}$ Department of Physics and Astronomy, University of Padua, Via Marzolo 8, 35131 Padua, Italy, \\ ${ }^{2}$ National Institute for Nuclear Physics (INFN), section Padua, Via Marzolo, 8, 35131 Padua, Italy, \\ ${ }^{3}$ National Laboratories of Legnaro (INFN), Viale dell Università 2, 35020 Legnaro (Pd), Italy, \\ 4 "Konstantin Preslavsky" University of Shumen, Faculty of Natural Sciences, Department of Theoretical and \\ Applied Physics, 115 Universitetska Str., 9712, Shumen, Bulgaria \\ e-mail: nikolay.uzunov@lnl.infn.it
}

\begin{abstract}
In our previous research we have observed that the fluorescence emission from water solutions of Single-Walled Carbon Nano-Tubes (SWCNT), excited by a laser with a wavelength of 830nm, diminishes with the time. We have already proved that such a fading is a function of the storage time and the storage temperature. In order to study the emission of the SWCNT as a function of these two parameters we have designed and realized a special measurement compartment with a cuvette holder where the SWCNT solutions can be measured and stored at a fixed constant temperature for periods of time as long as several weeks. To maintain the measurement setup under a constant temperature we have designed special experimental setup based on two Peltier cells with electronic temperature control.
\end{abstract}

Keywords: SWCNT, Single Walled Carbon Nanotubes, fluorescence, Peltier cell

\section{Introduction}

Nanomaterials with dimensions ranging from a nanometer up to several hundred nanometers are comparable with many biological macromolecules, such as enzymes, antibodies, DNA plasmid, etc. Compared to the same-substance normal size objects, nanomaterials exhibit different physical and chemical properties and open the prospective to a large field of research comprising physics chemistry, biology, technologies, etc. They offer new opportunities for biomedical research and applications in various areas including biology and medicine. Nanobiotechnology by means of chemical methods links physical with biological sciences in the development of new tools and platforms for the understanding of biological systems, disease diagnosis and medical treatment $[1,2,3]$. Among these nanomaterials, carbon nanotubes (CNTs) have attracted recently the scientific interest thanks to their unique physical, mechanical and chemical properties $[4,5,6,7,8]$. A carbon nanotube, although not produced directly from graphite, can be envisioned as hollow cylinder of graphite layers [9]. In particular, a single-walled carbon nanotube (SWCNT) is a graphene ${ }^{1}$ sheet rolled into a cylindrical shape so that the structure is quasi one-dimensional with axial symmetry, and in general exhibiting a spiral conformation, called chirality.

SWCNT can have a diameter of about 0.7-10.0 nm, though the most frequent experimentally observed ones are with diameters larger than $2 \mathrm{~nm}$. They can be considered as one-dimensional nanostructures if both ends ${ }^{2}$ of a carbon nanotube are neglected and the attention is focused on the large aspect ratio of the cylinder (i.e., length/diameter which can be as large as $10^{4}-10^{5}$ ) [10]. Another graphene configuration possibility is the multi-walled carbon nanotube (MWCNT) that is a tube comprising several, concentrically arranged single-

\footnotetext{
${ }^{1}$ Graphene is a monoatomic, two-dimensional layer of graphite

${ }^{2}$ The ends are often called caps and consist of "hemisphere" of fullerene; each cap is made with six five-membered carbon ring (pentagons) and an appropriate number of six-membered carbon ring (hexagons), that are placed properly in order to fit perfectly the section of the cylinder. 61
}

Corresponding author: nikolay.uzunov@lnl.infn.it DOI: 10.1515/asn-2017-0010 
walled carbon nanotubes. They have similar lengths to the single-walled tubes, but much larger diameters (the inner and the outer diameters are around 5 and $100 \mathrm{~nm}$, respectively, corresponding to 30 coaxial tubes) [9]. SWCNTs, thanks to their attractive and unparalleled physical properties including, but not limited, to electric conductance, high mechanical stiffness, light weight, transistor behavior, piezo-resistance, thermal conductivity, luminescence, electrochemical bond expansion and their versatile chemistry, as well as their size, shape and structure, have been studied for potential applications in biology [11]. As explained SWCNTs (and nanoparticle in general) have nano-dimensions of many orders of magnitude smaller than human cells, and therefore they can offer unprecedented interactions with biomolecules both on the surface and inside the cells, which may revolutionize disease treatments and diagnosis. Amongst the other very interesting properties it is the photoluminescence they exhibit in the near infrared (NIR) region when they are irradiated with visible light in the wavelength range up to $900 \mathrm{~nm}[12,13]$. Such kind of fluorescence is a matter of interest with respect to further biological applications such as deep tissue imaging. The transmission of NIR light in biological tissues is of the order of several millimetres. For small animals such as mice this is sufficient for imaging organs administered with SWCNTs.

In our previous research we have observed that the fluorescence emission from water solutions of SWCNT, excited by a laser with a wavelength of $830 \mathrm{~nm}$, diminishes with the time [14]. We have already proved that such a fading is a function of the storage time and the storage temperature. In order to study the emission of the SWCNT as a function of these two parameters we have designed and realized a special measurement compartment (thermostatic chamber) with a cuvette holder, where the SWCNT solutions can be measured and stored at a fixed constant temperature for periods of time as long as several weeks. In this article we present the design and realization of the cuvette compartment with the electronic modules to maintain the whole system in a constant temperature.

\section{Materials and Methods}

Measuring setup for the fluorescence emission of sodium cholate aqueous solution of SWCNT is compounded by the emission laser with a certain wavelength, a spectrometer for near-infrared (NIR) emission, a device to measure the intensity of the incoming laser beam and the intensity after the transmission in the sample, a cuvette holder with a system for measuring the fluorescence emission from the sample as well as a thermostatic system for maintenance and control of the temperature in the cuvette. All blocks are mounted on optical bank.

To characterize the fluorescence emission of SWCNTs it is necessary to implement a system capable of generating a laser light with the correct wavelength using a laser diode. In our setup we have implemented THORLABS laser diode M9-830-0150 emitting at $830 \mathrm{~nm}$ with maximum power output of $150 \mathrm{~mW}$. Measurements of the fluorescence spectrum of the SWCNT are carried out very close to the cuvette surface using InGaAs spectrometer Hamamatsu C9406GC. Additional measurements of the intensity of the laser beam before and after passing through the cuvette are conducting using a THORLABS PM100 USB powermeter. Since it was necessary to ensure constant temperature of the cuvette with the solution of SWCNT a small thermostatic chamber with a cuvette holder was designed. Two Peltier cells with air cooling were incorporated into the thermostatic chamber and a sensor for control of the temperature was mounted in it. To make manageable everything it was chosen to control these operations using a single computer. To do so, on a PC with Windows system have been installed management programs operating the NIR spectrometer and THORLABS powermeter. Moreover a software based on LabVIEW 8.2, which controls and maintains the temperature the temperature in the thermostatic chamber and records its values over time, was implemented. The software exploits a PID algorithm (Proportional, Integral, Derivative) [15] capable of controlling temperatures of a minimum of $5^{\circ} \mathrm{C}$ to a maximum of $50^{\circ} \mathrm{C}$. A suitable electronic, controlled by the PID, operates the current in the two Peltier cells installed on the outer surfaces of the chamber. In the same way another circuit, similar to the one described above, controls and maintains the temperature of the NIR spectrometer. In addition to this, to monitor the water condensation in the thermostatic chamber, it has been inserted a room temperature sensor and a humidity sensor. For the power supply and for the temperature maintenances of the laser source, however, were used dedicated devices: power supply block 205C THORLAB LDC and temperature control block THORLAB TED200C. The system, as a whole, has been

62

Corresponding author: nikolay.uzunov@lnl.infn.it

DOI: 10.1515/asn-2017-0010 
designed to be easily modified, where appropriate, supplemented by new parts, so as to respond to all the needs that would be created during the future research work.

\section{Experimental setup}

The whole measuring system is compounded by the following elements:

1. A PC which runs on software written in LabVIEW 8.2 by National Instruments

2. A plugged in a PC National Instruments board PCI 6025E that communicates with the hardware cards and temperature sensors.

3. Two constructed in the laboratory hardware cards on the basis matrix board composed by the 16F876A PIC microcontroller from Microchip, inside which there is a module for Pulse Width Modulation (PWM) [15], from the driver (a chip initialed Tc4469 of Microchip), and the MOSFETs (a IRLI 3705N of International Rectifier IOR able to drive the current in the Peltier cell). A board controls the temperature of the thermostatic chamber while the other controls the spectrometer.

4. Three temperature sensors LM35 TEXAS INSTRUMENTS, with corresponding signal amplifiers, one for thermostatic chamber, and the last one for spectrometer to room temperature.

5. Three power supplies TTi EL302RT used to power the hardware cards and the amplifiers of the temperature sensors

6. A thermostatic chamber made of aluminum, insulated with polystyrene of $1 \mathrm{~cm}$ and provided with two Peltier cells (Global Component Sourcing ET-127-14-15) complete with heat sink and fan.

7. A humidity sensor Honeywell HIH-5030 with its power supply

The block diagram in Fig. 1 shows the interaction of the functional blocks of the system with their connections. The red connections represent the supply lines, celestial lines indicates sensor signals, the yellow ones are the amplified signals from the temperature sensors and also those of the two control hardware cards while the orange links represent the currents of the Peltier cell signals.

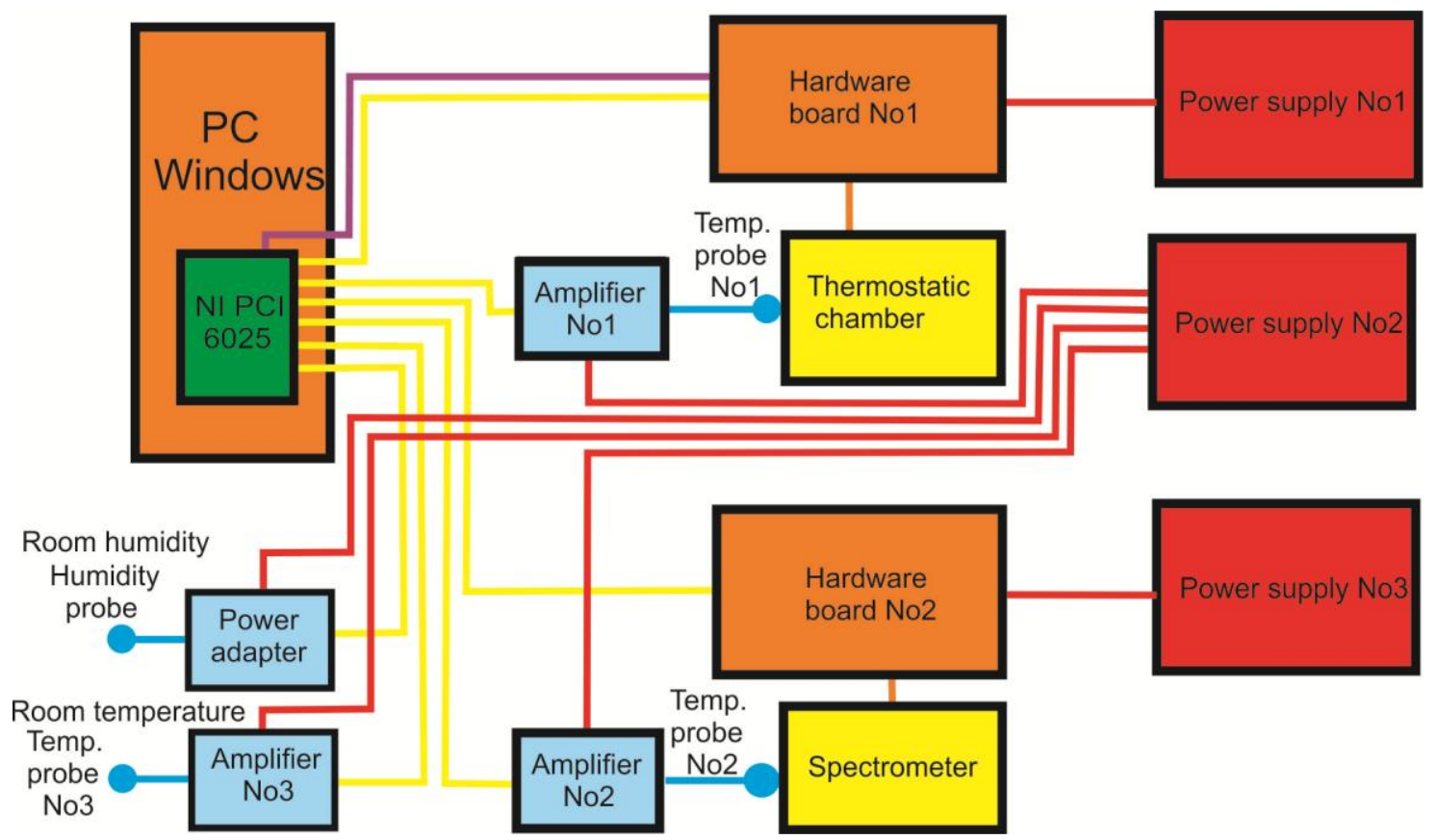

Fig. 1. Block diagram of the electronic setup for long-time constant-temperature maintenance of the thermostatic chamber

The whole system is controlled by a software written in LabVIEW 8.2, which allows to read the three temperature sensors and the humidity through one 12-bit ADC (present in NIPCI6025 tab), to operate the

63

Corresponding author: nikolay.uzunov@lnl.infn.it

DOI: 10.1515/asn-2017-0010 
hardware circuits by means of the DAC output channels 12 bits of NIPCI 6025, to handle the two PID temperature controls, to save the acquired values of the temperature and humidity versus time in a file in excel format, and to visualize graphs of the trend in run-time values.

The program is formed by a continuous loop which is executed every $100 \mathrm{~ms}$, a time more than sufficient to properly control the slow variations of the temperature. Before running the loop the program makes a variety of settings related to the initial conditions of the variables involved and also sets the analog channels and the name and location of the data files. Inside the loop the temperature and humidity sensors are read, are calculated also the variables of the two PID controls, and are updated their output variables, then the data are saved you in a file and after that the graphics in the front panel are displayed. After the loop, which can only be closed by the front panel, the settings of the analog channels are reset and the data is closed. A second loop in parallel manages the reading of the button which imposes heating or cooling the thermostatic chamber, depending on the temperature value to be maintained. These two modes are implemented through the switching of a relay that reverses the current in the Peltier cells.

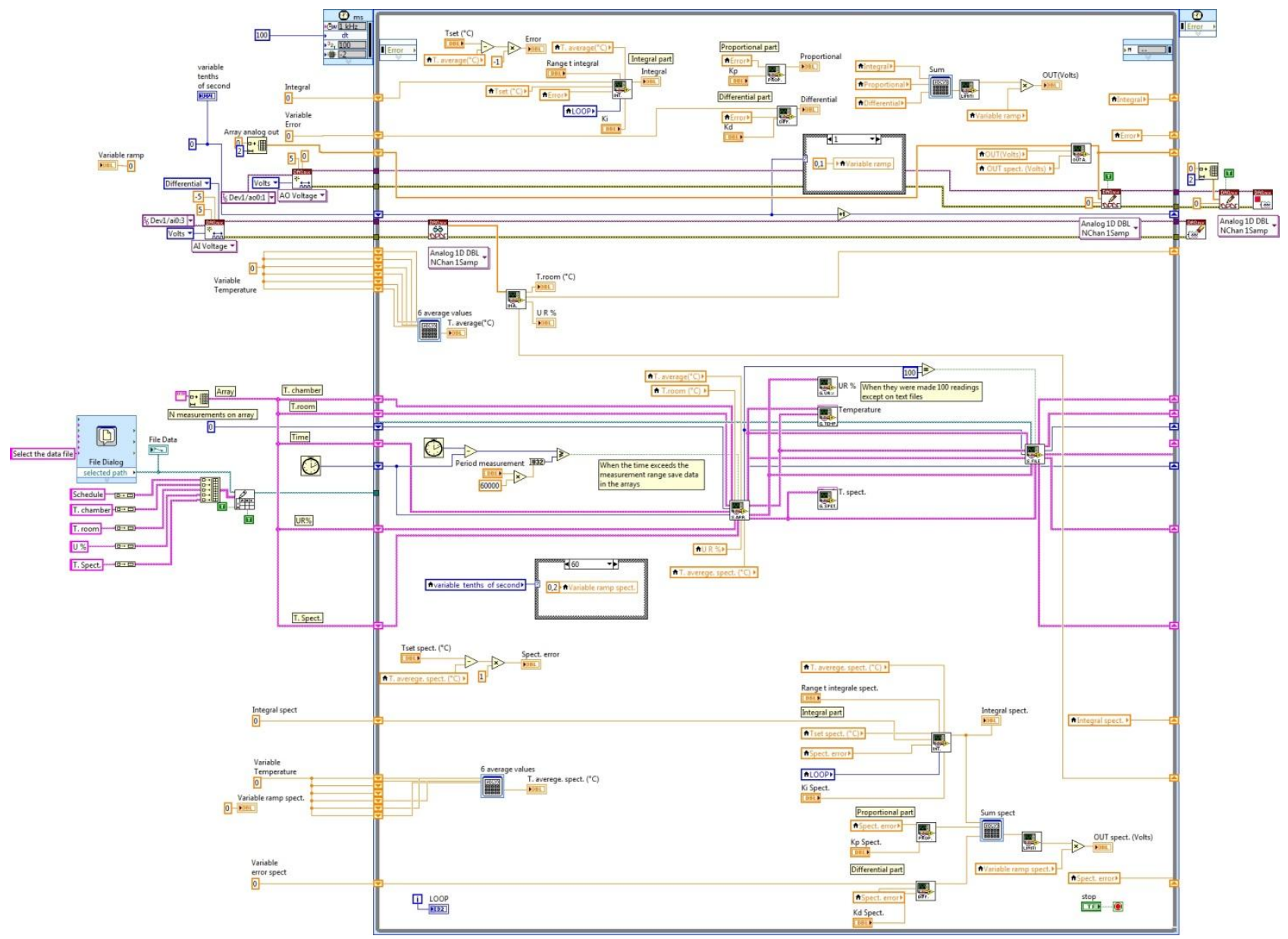

Fig. 2. Block diagram of the software for temperature and humidity control and regulation The National Instruments board NI PCI 6025 E

This card through the PCI bus of the PC allows us to interface the analog and digital signals from the outside with the LabVIEW software. It has the ability to manage different signals: up to 8 differential analog inputs 12-bit, two 12-bit analog outputs, 32 digital inputs/outputs, and two 24-bit counters/timers. It uses a single ADC with sampling/rate maximum of $200 \mathrm{Ks} / \mathrm{s}$ and a DAC with sampling/rate maximum of $10 \mathrm{ks} / \mathrm{s}$.

For our application we have used 4 differential analog inputs (temperature and humidity sensors), two analog outputs (control hardware cards) and a digital signal (control of the relay, inversion of the current in Peltier cells for the thermostatic chamber).

64 
Hardware board for control of the temperature of the thermostatic chamber and of the NIR spectrometer Two hardware boards (Fig. 3) have been constructed in our laboratory to command adequately the current in the Peltier cells of the thermostatic chamber and to control the temperature of the spectrometer. The construction of these cards has been necessary both to be able to control, at the hardware level, the cell current in PWM (Pulse Width Modulation), and to eliminate the problems of thermal dissipation in the power consuming MOS-FET element as well as to disengage from the need to generate the PWM signal directly from the LabVIEW software, already engaged in various other processing. The electronic circuit is formed by three main blocks: a PIC16F876A microcontroller, an integrated driver Tc4469 and a MOS-FET IRLI3705N. The microcontroller receives the analog signal from the National NI6025 tab, translates it into PWM signal and sends it to the integrated driver. The Tc4469 amplifies it first in a current and transmits it to the gate of the MOS-FET which can drive with a further amplification the current of the Peltier cells. The cards are both powered by a voltage of 17 Volts with a maximum current of $2.5 \mathrm{~A}$.
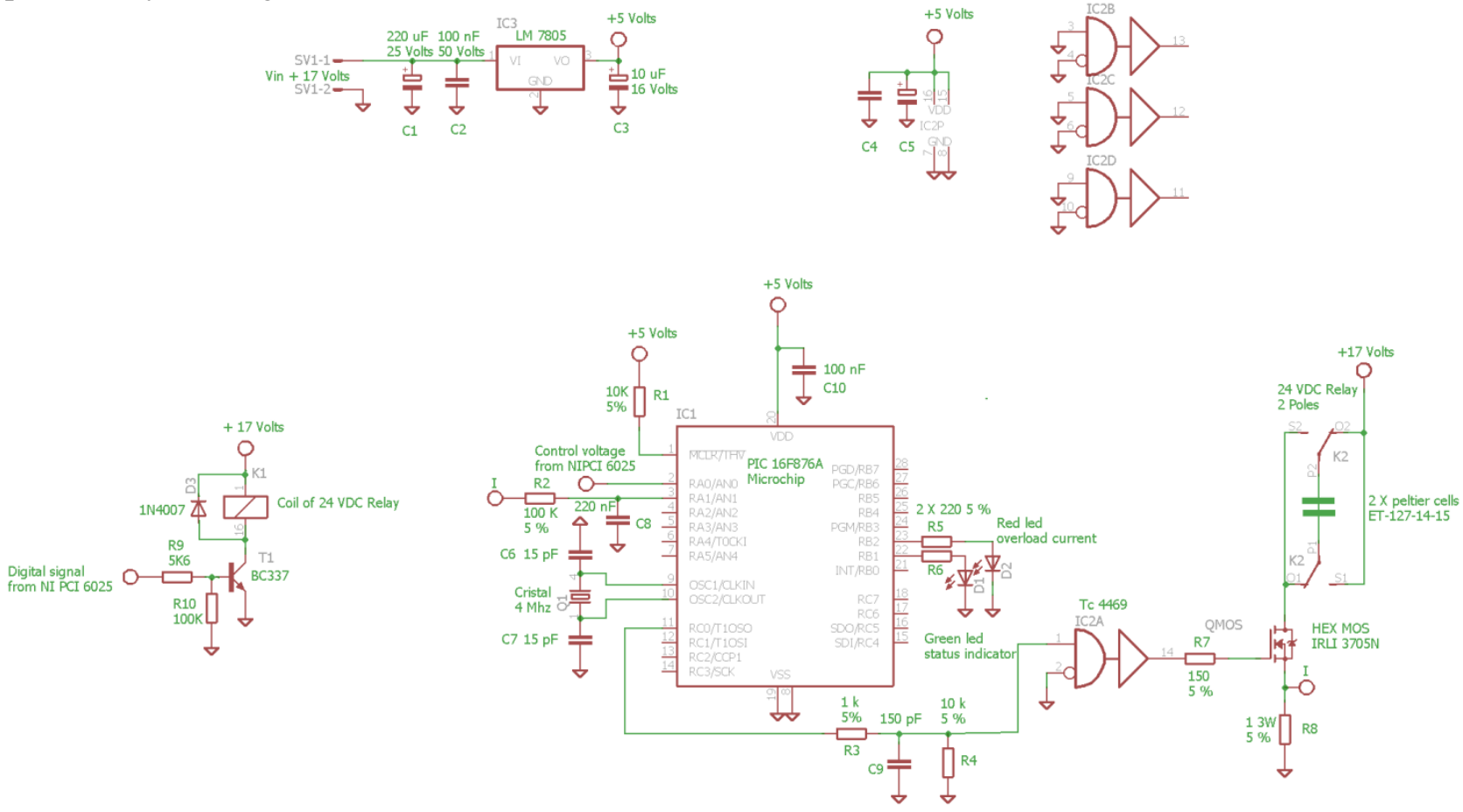

Fig. 3. The electrical circuit schemes of the hardware cards Amplifiers of the temperature sensor signals and the humidity sensor power supply circuit

The complete system uses three integrated temperature sensors LM35 of Texas Instruments. These devices offer an accuracy of $+/-0.4^{\circ} \mathrm{C}$ throughout the operation range of -55 to $150^{\circ} \mathrm{C}$, but for the range of temperature of use of our system (from 5 to $50^{\circ} \mathrm{C}$ ) the typical error is reduced to a maximum of $+/-0.1^{\circ} \mathrm{C}$, that is more than sufficient for our application. This type of sensors generates an output voltage of 10 millivolts per degree of temperature. Having to measure also temperatures of the order of a few degrees, hence corresponding to very low output voltages, it was necessary to amplify the signal by a factor of 10 . The amplification was carried out with an instrumentation amplifier INA128 of Burr Brown, with a CMR of 120 $\mathrm{db}$, possessing a low offset and a drift offset of $0.5 \mu \mathrm{Volts} /{ }^{\circ} \mathrm{C}$. The amplification is fed by dual voltage of +10 and -10 volts with a maximum current of $100 \mathrm{~mA}$ (Fig. 4).

65 


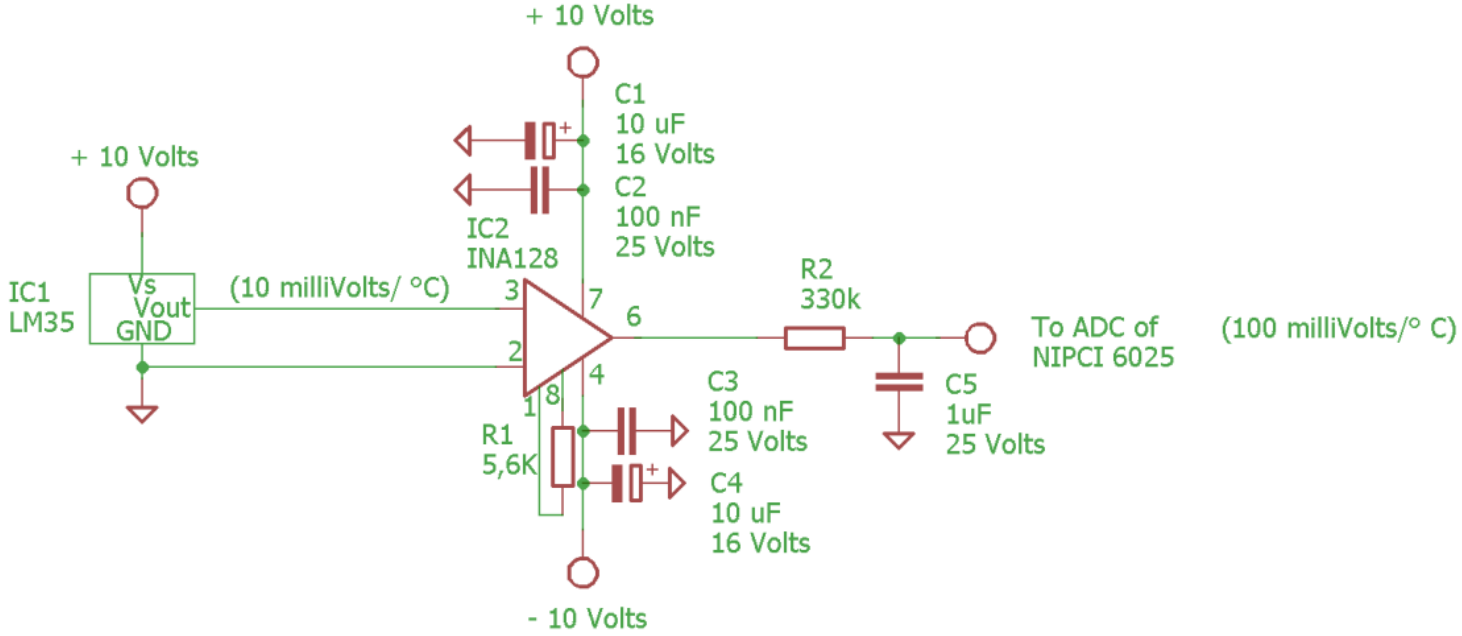

Fig 4. Electrical scheme of the amplifier circuit

For the humidity sensor, Honeywell HIH-5030 we used an application circuit (Fig. 5) recommended by the manufacturer. It was chosen to supply the sensor to 3.3 Volts which is the typical voltage with which have been obtained most of the characteristic curves included in the technical data-sheet. The 3.3 Volts have been obtained from an LM317 of the National Semiconductor, a regulator capable of generating stabilized voltages from 1.2 Volts to 40 Volts. The output of the sensor in volts is corrected, in the LabVIEW program from an equation which takes account of its behavior as a function of the supply voltage. The relative humidity is obtained then through the formula $\mathrm{HR}=($ Vsupply $)(0.00636$ (Vout sensor $)+0.1515)$. AT the end this circuit allows to measure the relative humidity from $11 \%$ to $89 \%$ with a maximum accuracy of $+/-3 \%$. The power supply of the moisture sensor is fed from the outside from $+10 \mathrm{~V}$ with a maximum current of $100 \mathrm{~mA}$.
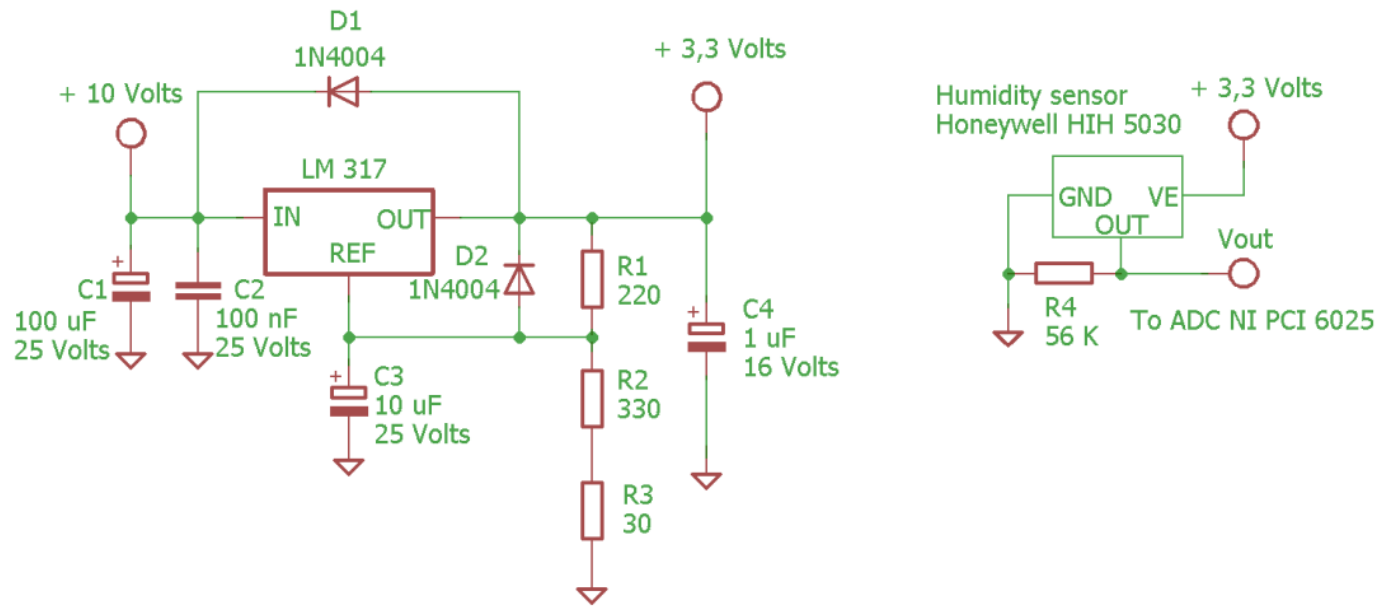

Fig 5. The power supply circuit diagram of the humidity sensor

66 

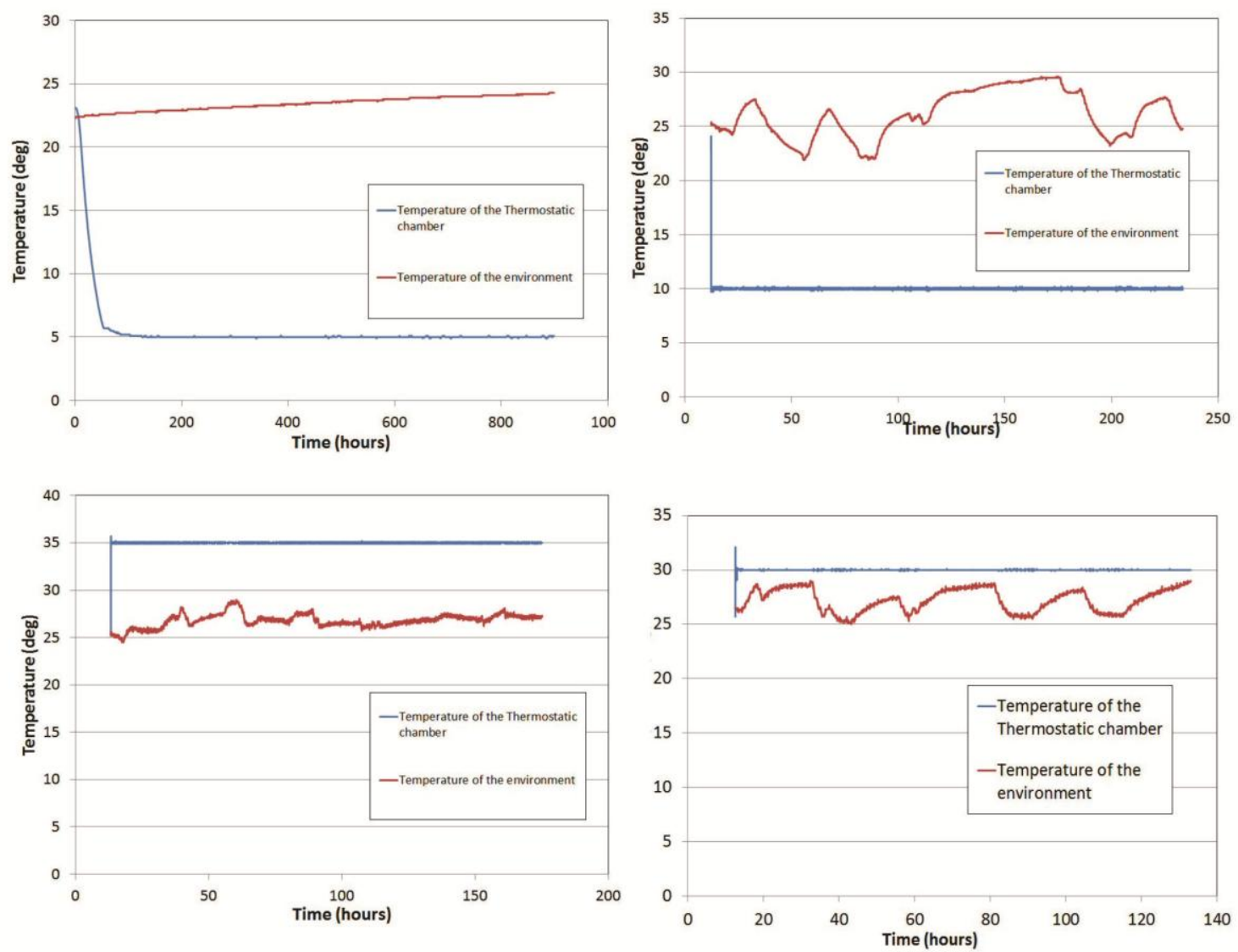

Fig 6. Temperature of the thermostatic chamber as a function of the storage time for 4 different storage temperatures (blue lines). The temperature of the air in the laboratory is shown in red.

\section{Results}

The long-time constant-temperature maintenance (up to twenty days) of a cuvette filled with solution of Single-Walled Carbon Nano-Tubes was tested for several temperatures beginning with $5^{\circ} \mathrm{C}$ to $35^{\circ} \mathrm{C}$ with a step of $5^{\circ} \mathrm{C}$. The humidity of the measuring hall was also controlled and always maintained low enough to prevent water condensation of the cuvette. Thus measurements of the fluorescence emissions were also possible to be carried out. Figure 6 shows time plots of four temperatures of the thermostatic chamber: $5^{\circ} \mathrm{C}, 10^{\circ} \mathrm{C}, 30^{\circ} \mathrm{C}$ and $35^{\circ} \mathrm{C}$. The temperature of the environment is also shown in the figure to give an idea about the conditions of the measuring hall. One can clearly see the rapid temperature change of the thermostatic chamber in the beginning of each plot, and its smooth horizontal-line part after the setup temperature is achieved.

Table 1 Measurements of the average temperature in the thermostatic chamber with the cuvette holder for several storage-time temperatures

\begin{tabular}{ccccc}
\hline $\begin{array}{c}\text { Storage } \\
\text { temperature set } \\
\left({ }^{0} \mathrm{C}\right)\end{array}$ & $\begin{array}{c}\text { Average } \\
\text { temperature } \\
\left({ }^{0} \mathrm{C}\right)\end{array}$ & $\begin{array}{c}\text { Standard deviation } \\
\left({ }^{0} \mathrm{C}\right)\end{array}$ & $\begin{array}{c}\text { Minimum } \\
\text { temperature } \\
\text { measured } \\
\left({ }^{0} \mathrm{C}\right)\end{array}$ & $\begin{array}{c}\text { Maximum } \\
\text { temperature } \\
\text { measured } \\
\left({ }^{0} \mathrm{C}\right)\end{array}$ \\
\hline 5 & 5.0088 & 0.035 & 4.9 & 5.1 \\
10 & 10.0009 & 0.057 & 9.7 & 10.2 \\
15 & 14.9996 & 0.0155 & 14.9 & 15.1
\end{tabular}

67

Corresponding author: nikolay.uzunov@lnl.infn.it DOI: 10.1515/asn-2017-0010 


\begin{tabular}{ccccc}
20 & 19.9997 & 0.0237 & 18.8 & 21.1 \\
25 & 25.0552 & 0.3765 & 24.8 & 26.7 \\
30 & 30.0013 & 0.0620 & 29 & 32.1 \\
35 & 35.001 & 0.0391 & 34.4 & 35.7 \\
\hline
\end{tabular}

Table 1 demonstrates the results from the measurements of the temperature in the thermostatic chamber with the cuvette holder. The storage time for each of the temperatures maintained in the chamber exceeded 10 days. The temperature measurements were made in intervals of 6 seconds and were registered by the computer. Thus the values shown in Table 1 are averaged values from a large number of temperature measurements.

\section{Conclusions}

We have designed and constructed a setup for fluorescence emission measurements and long-time constanttemperature maintenance of Single-Walled Carbon Nano-Tubes in water solutions. The whole setup was mounted and tested in the Laboratory for Radiopharmaceuticals and Molecular Imaging (LARIM) at the National Laboratories of Legnaro, INFN, Italy.

The specific conditions for constant temperature maintenance of the measuring thermostatic chamber for periods up to one month in the laboratory required a very thorough approach in the search and implementation of the electronic blocks and circuits. Moreover, the special requirements for stability, reliability and fire safety as well as the requirements for protection against overloads and short circuits necessitated the construction of a system with much more specific capacities and capabilities. Realization of such a system was possible due to the opportunities offered by the use of specialized boards such as the National Instruments board NI PCI $6025 \mathrm{E}$ and moreover the possibility to control and operate all the processes using an appropriate software written in LabVIEW 8.2.

Real time measurement tests with this system have already been carried out so far at several storage temperatures. The system proved to possess excellent long-time storage-temperature stability and reliability and is suitable to conduct the fluorescence emission studies of different solutions of SWCNT.

\section{Acknowledgment}

This research was partially supported by the Project RD 10-399/07.03.2016 of the University of Shumen, Shumen, Bulgaria.

\section{References}

[1] Whitesides G. M., The "right" size in nanobiotechnology, Nat Biotech, 2003, 21: 1161 - 1165.

[2] Lowe C. R., Nanobiotechnology: The fabrication and applications of chemical and biological nanostructures, Curr Opin Chem Biol, 2000, 10: 428 - 434.

[3] Wang L., Zhao W., Tan W., Bioconjugated silica nanoparticles: Development and applications, Nano Res., 2008, 1: 99 - 115.

[4] Iijima S., Helical microtubules of graphitic carbon, Nature, 1991, 354: 56 - 58

[5] Dai H., Carbon nanotubes: Synthesis, integration, and properties, Acc Chem Res, 2002, 35: 1035 - 1044.

[6] Dresselhaus M., Dai H., Special Issue on Carbon Nanotube, MRS bull., 2004 Vol. 29. 2004.

[7] Golberg D., Costa P.M.F.J., Mitome M., Bando Y., Nanotubes in a gradient electric field as revealed by STM-TEM technique, Nano Res, 2008, 1: 166 - 175.

[8] Zhou W., Rutherglen C., Burke P., Wafer scale synthesis of dense aligned arrays of single walled carbon nanotubes, Nano Res., 2008, 1: 158 - 165.

[9] Reich S., Thomsean C., Maultzsch J., Carbon Nanotubes: Basic Concepts and Physical Properties, WileyVCH Verlag, Weinheim, 2004.

[10] Saito R., Dresselhaus G., Dresselhaus M. S., Physical Properties of Carbon Nanotubes, Imperial College Press, London, 1998.

68 
[11] Liu Z., Tabakman S., Welsher K., Dai H., Carbon Nanotubes in Biology and Medicine: In vitro and in vivo Detection, Imaging and Drug delivery, Nano Res., 2009, 1; 2(2): 85 - 120.

[12] Welsher K., Liu Z., Daranciang D., Dai H., Selective probing and imaging of cells with single walled carbon nanotubes as near-infrared fluorescent molecules, Nano Lett. 2008, 8, 2: 586 - 590.

[13] Murakami Y, Junichiro K., Nonlinear Photoluminescence Excitation Spectroscopy of Carbon Nanotubes: Exploring the Upper Density Limit of One-Dimensional Excitons, Phys. Rev. Lett., 2009, 102: 0374011 - 4 [14] Uzunov N. M., Atroshchenko K., Fontana C. L., Bello M., Rosato A., Melendez-Alafort L., Manov L., Menna E., Salice P., Moschini G., Study of Photoluminescence Properties of Single-Walled Carbon Nanotubes, INFN- LNL Report 239, 2013, 130 - 131

[15] Hart D., Power Electronics. McGraw - HIll. (2010). 